\title{
Analysis of performance degradation in sleep-mode enabled core optical networks
}

\author{
Albert Pagès, Massimo Tornatore, Jordi Perelló, Salvatore Spadaro and Annalisa Morea
}

\begin{abstract}
Energy efficiency is an important target for the management of Wavelength Division Multiplexing-based core optical networks. A possible power management strategy for energy efficient optical networks consists in putting opto-electronic devices (transponders and regenerators) into a low power mode, called sleep or idle, or even turning them off, during low traffic periods. It has been shown that sleepmode enabled transponders and regenerators yield to substantial energy savings; however, their non-negligible wakeup time may degrade the network performance in terms of blocking probability of the connections. Opaque networks, where transponders are utilized at each node in the endto-end path, are specially prone to such degradations since multiple network nodes may exhaust simultaneously their pool of transponders due to long wake-up times, increasing the blocking probability. On the other hand, these degradations are less severe in transparent networks. In this paper, we evaluate how the duration of the wake-up time affects the performance of the network in terms of blocking probability depending on its architecture (transparent, translucent or opaque) and the dynamicity of the traffic. Additionally, we propose a novel routing algorithm to mitigate the blocking probability due to the wake-up time. The benefits of the proposed algorithm are highlighted through extensive results.
\end{abstract}

Index Terms-Energy efficiency; On-Off strategies; Routing algorithms.

\section{INTRODUCTION}

$\mathbf{T}$ ODAY'S access networks represent around $70 \%$ of the overall power consumption of telecommunications networks, while core networks represent about $15 \%$ of it. However as the traffic on Internet increases, it is forecast that the power consumption in telecommunications networks will almost double by 2017 , with access and core networks accounting for around $38 \%$ and $42 \%$ of such power consumption, respectively [1]. For these reasons, it is of capital importance to reduce the power consumption in also core networks. Wavelength Division Multiplexing (WDM)-based optical networks have helped reducing the power consumption of core networks by allowing large amounts of traffic to bypass routers through optical switching [2], [3]. However, additional energy savings in core optical networks can be achieved by using more energy-efficient opto-electronic (OE) devices. In [4], authors presented a novel architecture for both transponders (TSPs) and regenerators (REGs) capable of putting some of their modules either in a low-power consumption mode (idle mode), or turning them off (off mode) when they do not support traffic. OE devices modules

Manuscript received July 1, 2014.

A. Pagès, J. Perelló and S. Spadaro are with the Advanced Broadband Communications Center (CCABA), Universitat Politècnica de Catalunya (UPC), Barcelona, Spain (e-mail: albertpages@tsc.upc.edu, perello@ac.upc.edu, spadaro@tsc.upc.edu).

M. Tornatore is with the Department of Electronics and Information (DEIB), Politecnico di Milano, Milan, Italy (e-mail: tornator@elet.polimi.it).

A. Morea is with Alcatel Lucent, Bell Labs, Nozay, France (e-mail: annalisa.morea@alcatel-lucent.com)

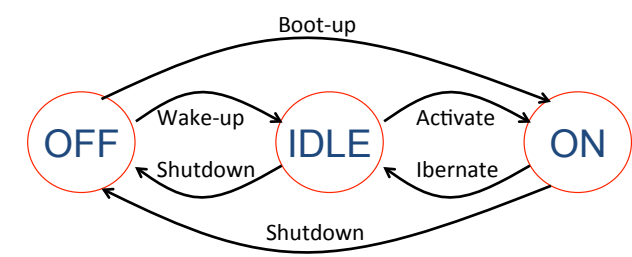

Fig. 1. Diagram of the transitions between the three power states of a power adaptive $\mathrm{OE}$ device.

can be then turned back in a fully operative (on) mode whenever new connections are established. The dynamic management of the $\mathrm{OE}$ devices power states is carried on according to the current traffic load and to the requirements in terms of connection set-up time. Fig. 1 depicts the interstate transitions for such power adaptive devices.

With such a power management capabilities, the authors in [4] demonstrated that up to $56 \%$ power savings can be attained with respect to traditional WDM networks, where devices are always powered-on regardless if they are transmitting data or not. Other studies (e.g. [5], [6]) confirm the benefits of putting some elements in core optical networks into a low-power consumption mode. In [7], different classes of traffic are considered and, as a consequence, the setup time of the connections becomes a critical parameter, since typically high-priority traffic (e.g., real-time traffic) requires short set-up times, while low-priority traffic does not. Considering that the boot-up time (i.e., the time required for the transition of a TSP or a REG from the off to on mode) can be quite long (tens of seconds [4]), available TSPs and REGs in the off mode cannot be allocated to support highpriority traffic.

Therefore, TSPs and REGs in the off mode should only be allocated to low-priority traffic, while TSPs and REGs in idle mode should be used to allocate high-priority traffic, since idle devices have a much lower activation time (time required for the transition from the idle to on mode) that can match the more stringent set-up time requirements. Note that, by reserving some of the available devices per node for high-priority traffic and putting them into idle state, it is possible to improve the overall reactivity of the network, satisfying both high- and low-priority connections requirements, and, at the same time, reduce the power consumption of the network.

Although there are works that analyzed the blocking probability in sleep-mode enabled optical networks with lowand high-priority traffic, such as in [8], there are no works, to the best of our knowledge, that evaluate the impact of the OE devices wake-up time on the overall network blocking probability (especially for high-priority traffic) in core optical networks. Essentially, depending on the duration of the TSP/REG wake-up process, and the arrival rate of the connections, may occur some situation where devices in idle 
state may not be available to be allocated to incoming highpriority connections.

Therefore, a proper dimensioning of the number of devices in idle mode per node to support high-priority traffic must be done. In [9], we evaluated the impact of TSPs and REGs wake-up time in a translucent optical network in terms of blocking probability of the connections as a function of the traffic dynamicity, i.e. the arrival rate and the duration of the connections. Under the same load conditions, a more dynamic traffic implies that the arrival/departure rate of the connections is faster than in less dynamic traffic, with connections lasting less and arriving at a higher rate. Under such conditions, higher traffic dynamicities may suffer from higher blocking due to non-negligible wake-up times, since more connections may arrive at a network node during a wake-up operation. Thus, we found that, although short wake-up times do not entail significant performance degradations even for high dynamic traffic, as the duration of the wake-up time grows, less and less dynamic traffic can be supported without incurring into severe performance degradations. For instance, for a wake-up time of $1 \mathrm{~min}$., the blocking probability increased by a factor of 14 when compared against an ideal case (wake-up time $=0$ ) for low traffic dynamicities, while it increased by a factor of almost 140 for higher traffic dynamicities.

In light of these results, it becomes clear that the proper dimensioning of the idle resources reserved for high-priority is tightly related to the traffic dynamicity and the duration of the wake-up operation of the devices. To this end, in [9] we also analyzed the necessary extra idle resources per node needed to achieve sustainable blocking figures. We found that it is possible to compensate the effects of non-negligible wake-up times with a small increase (3-6\%) on the number of reserved resources per node for high-priority traffic, even for high traffic dynamicities. The required increase in the number of idle resources translates to a marginal increase on the average daily power consumption (around 1.3\%), since the additional devices enjoy the benefit of the low power consumption of the idle mode when not in use. Moreover, despite the slight increase in the energy consumption, average power savings around $65 \%$ were found compared to a traditional WDM optical network, where devices stay always poweredon regardless if they are sending/receiving traffic or not.

However, an open issue is how this wake-up operation affects different network architectures of core optical networks, namely: 1) opaque optical networks, where connections undergo an Optical-Electrical-Optical (O-E-O) conversion in every intermediate node along the end-to-end path; 2) translucent optical networks, where connections may undergo a regeneration process on some of the nodes along the end-to-end path; and 3) transparent optical networks, where connections always remain into the optical domain along the entire end-to-end path. Due to the different number of devices that may undergo a wake-up operation in these three types of optical networks significant differences in terms of blocking associated to the wake-up time can arise.

To this end, in this work we evaluate the degradation on the overall network performance in terms of blocking probability due to the wake-up time of the devices in a core optical network on all the three architectures. Moreover, the evaluation of the power savings with respect to traditional WDM scenarios is also performed. Additionally, we introduce a novel routing algorithm called Wake-up Time Aware Rout- ing (WTAR) that aims to compensate the blocking due to the presence of non-negligible wake-up times.

The rest of the paper is structured as follows: Section II describes the employed resource reservation strategy and the proposed WTAR algorithm. Section III depicts the evaluation scenario and the results obtained during the performance evaluation. Finally, Section IV draws up the main conclusions.

\section{TSPS AND REGS ALLOCATION STRATEGY}

In this paper, we consider the dynamic management of a core network, where connections arrive and disconnect at random to/from the network. Connections in such situation are understood as the aggregation at source node of the core network of multiple client connections coming from the same access/metro network that are going to the same destination access/metro network. Future core optical networks are expected to be more dynamically operated than nowadays [10], mainly due to the continuous traffic growth of Internet and new emerging applications, such as video on demand and multimedia social networks, which require a more flexible resource allocation in order to satisfy their needs. In such scenario, it becomes highly important to keep the associated operational expenditures (OPEX) of the network at low levels, so as to cope with these new requirements in an efficient way. A very important parameter in this regard is the power consumption of the network. Thus, it is possible to save costs by adjusting the number of active devices according to the current traffic.

Additionally, future networks are predicted to be more service aware in order to cope with the heterogeneity of the applications that will run on top of them. Technologies such as deep packet inspection [11] will allow network operators to classify traffic in multiple categories, for instance, high priority traffic coming from financial applications or telemedicine, which require a high Quality of Service (QoS), and low priority traffic with less stringent QoS. By treating the traffic according to their priority, it is predicted that network operators will see an increase in their revenues

For this reasons, in this paper we are targeting the dynamic management of the power states of $\mathrm{OE}$ devices in a core optical network, considering that multiple classes of traffic coexist. To this end, we consider two classes of connections, namely, high-priority and low-priority connections, characterized by two different requirements in terms of set-up time. Specifically, we assume that the high-priority connections have more stringent set-up time requirements than low-priority connections. To manage the establishment of the high-priority connections according to their set-up time target, a possible energy-efficient strategy consists on reserving part of the node OE resources (TSPs and REGs) for high-priority traffic, setting them in idle mode for a prompt establishment of such connections. Assuming that every node in the network is equipped with exactly $N$ TSPs and $N^{\prime}$ REGs, a fraction of such resources ( $m$ and $m^{\prime}$, respectively) are set in idle state and will be exploited by high-priority traffic. Note that both values $N$ and $N^{\prime}$ as well as $m$ and $m^{\prime}$ are the same for all nodes and fixed a priori. Then, the rest of the unused resources (those that are neither transmitting/receiving data nor reserved for highpriority traffic) are left in off mode and used for future lowpriority incoming connections or to be switched to idle when $m$ or $m^{\prime}$ decreases. 


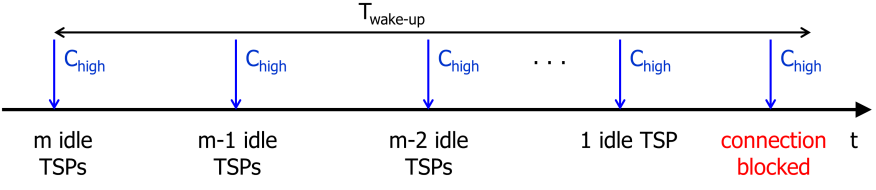

Fig. 2. Example of blocking of high-priority connections due to the influence of the wake-up time.

In order to maintain the pool of resources reserved for high-priority traffic, every time an idle device (either a TSP or REG) is allocated for a new high-priority connection (and thus set to on mode), if and only if spare off devices exist, a wake-up operation will be triggered and an off device will start changing to idle mode. Hence, high-priority connections are blocked if no idle devices are available. As for lowpriority connections, if no off devices are available when they arrive, they are also blocked, since the devices in idle state are reserved only for high-priority connections. Additionally, when a high- or low-priority connection is torn-down, if the number of idle devices is lower than $m$ (TSPs) or $m^{\prime}$ (REGs), the released devices are hibernated to idle mode. Otherwise, the released devices are completely shut down (off mode).

Regarding the wake-up operation (transition from off to idle), its duration can dramatically affect the blocking levels on the network, specifically for the high-priority traffic. In this regard, high-priority connections can be blocked mainly for two reasons: 1) lack of free resources (TSPs, REGs or wavelengths) due to connections competing for them; or 2) lack of idle devices (TSPs or REGs) due to wake-up time delays.

As explained above, after an idle device is selected to support an incoming high-priority connection, a wake-up operation is also triggered to maintain the pool of reserved resources for high-priority traffic (a spare off device is set to idle). But, in case that additional $m-1\left(m^{\prime}-1\right)$ high-priority connections have to be established at that particular node during the wake-up operation, all idle devices will become exhausted. So, any further high-priority connection arriving before the ending of the wake-up operation will be blocked due to the lack of idle devices. Fig. 2 depicts an example of this event.

Some studies suggest that, with nowadays technologies, the duration of the wake-up time is on the order of tens of seconds (e.g. [4]). Depending on the inter-arrival rate of the connections, it may happen that the duration of the wake-up time is excessive, leading to unsustainable levels of blocking for network operators. This is specially critical in opaque optical networks, as they require the utilization of a TSP at every node on the end-to-end path. In this situation, a significant number of nodes may simultaneously exhaust their pool of reserved resources for high priority connections. For this reason, this kind of opaque network is more sensitive to performance degradations due to nonnegligible wake-up times. On the other hand, this situation is less severe in transparent networks, since the source and the destination nodes are the only ones affected by the lack of idle resources, hence less nodes may simultaneously run out of idle devices. Translucent optical networks represent an intermediate case due to the use of some regenerators (that are also affected by the wake-up time) along the endto-end path.

To reduce such blocking situations, we propose the novel
WTAR algorithm, that considers as an input the available idle resources in the end-to-end paths in the network and tries to balance the connections to avoid the exhaustion of idle devices in the nodes. In the following, we provide the details of two versions of the algorithm, one for the opaque case and another for the translucent case. We did not consider the transparent case since in this case the wake-up time only affects the source and the destination of the connections, hence there is no chance to avoid the exhaustion of idle devices in the nodes by means of balancing the connections.

\section{A. Opaque WTAR}

As we mentioned before, opaque networks are more sensitive to non-negligible wake-up times due to the fact that connections established in the network employ $2 \times n_{\text {hops }}$ TSPs, being $n_{\text {hops }}$ the number of links belonging to the end-to-end path. As the duration of the wake-up operation increases, the chances of running out of idle TSPs in a node increases, leading to blocking situations. At this point it is worth mentioning that we are considering an opaque network where nodes are equipped with $W$ TSPs per incoming/outgoing link (TSPs are considered bidirectional).

A possible strategy to avoid this problem is to encourage the load balancing of the idle devices on the network, reducing the congestion of the nodes, hence avoiding the blocking of a high priority connection due to the lack of free resources. Moreover, it is also interesting to balance the load of off devices since they will be employed to perform wakeup operations when needed. To this end, we can use the following path metrics:

$$
\begin{gathered}
\alpha \frac{l_{p}}{D_{r}}+(1-\alpha) \frac{m}{s_{i d l e, p}} \\
\alpha \frac{l_{p}}{D_{r}}+(1-\alpha) \frac{N-m}{s_{\text {off }, p}}
\end{gathered}
$$

where $l_{p}$ represents the length of the path and $D_{r}$ the transmission reach of the signals (both in $\mathrm{km}$.), $N$ and $m$ represent the total number of TSPs and the number of idle TSPs per node and bidirectional link (we remind the reader that the assumed node architecture has a dedicated set of TSPs for every incoming/outgoing link) reserved for high-priority connections, respectively, and $s_{i d l e, p}$ and $s_{o f f, p}$ represent the number of idle and off TSPs in the most congested node along the path, respectively. Note that the metrics are for the whole path, discriminating the candidate paths in terms of total length and most congested resources. Parameter $\alpha$ is a real value in $[0,1]$ and is used to set the emphasis of the routing policy. For instance, in the case of $\alpha=0$, the routing algorithm chooses as candidate paths the ones having larger values of $s_{i d l e, p}$ or $s_{o f f, p}$, depending on the priority. That is, it chooses the paths that have the least congested nodes, avoiding blocking situations due to the lack of resources in the nodes. However, since the connections are balanced in the network, longer paths might be chosen, thus increasing the number of TSPs that will be powered-on and, hence, the overall power consumption of the network.

On the other hand, if $\alpha=1$, shorter paths in terms of physical distance will be preferred as candidate paths. In this situation, the energy consumption is kept low, since less resources are powered-on. However, the availability of both idle and off TSPs will be not balanced and the blocking 
associated to the wake-up time will be higher. Scenarios with $\alpha$ between 0 and 1 represent a routing policy offering a tradeoff between power consumption and blocking probability.

Thus, the proposed WTAR algorithm can be described as follows:

1) When a connection arrives, calculate the first $K$ shortest paths between source and destination (where $K$ is an input parameter) utilizing the metric (1a) for a high-priority connection, or (1b) for a low-priority connection. In both cases, if 2 or more of the calculated paths have the same weight, the path with the lowest number of hops is prioritized.

2) Select the first path as the candidate path and try to establish the connection employing a First Fit (FF) criteria for the wavelength selection, considering that, thanks to the O-E-O capability of the nodes, the wavelength continuity constraint may be relaxed and different wavelengths along the path may be used. Remember that high-priority connections can only be served employing idle TSPs while low-priority connections can only be served employing off TSPs.

3) If the connection can be set-up along the selected path, mark the connection as successful. Otherwise, try the following path in the set.

4) If all the paths in the set have been explored and none has free resources, the connection is blocked.

\section{B. Translucent WTAR}

The WTAR algorithm for the translucent case presents slight differences from the one presented for the opaque case. In a translucent optical network, the connection stays always in the optical domain until regeneration is needed, which is performed by a REG at one of the regeneration pools of the network. In this case the REGs become the most critical resources, as they may be utilized in multiple points along the end-to-end path. As for add/drop TSPs, since they are only employed in the source and the destination nodes of the connection, there is no chance to balance their use by means of an appropriate routing algorithm. For these reasons, we develop a metric to help balancing the use of the REGs in the network similarly to how TSPs were balanced in the opaque WTAR case. Specifically:

$$
\begin{gathered}
\alpha \frac{l_{p}}{D_{r}}+(1-\alpha) \frac{m^{\prime}}{r_{i d l e, p}} \\
\alpha \frac{l_{p}}{D_{r}}+(1-\alpha) \frac{N^{\prime}-m^{\prime}}{r_{o f f, p}}
\end{gathered}
$$

where $N^{\prime}$ and $m^{\prime}$ represent the total number of REGs and the number of idle REGs reserved per node for high-priority connections, respectively, and $r_{i d l e, p}$ and $r_{o f f, p}$ represent the number of idle and off REGs in the most congested node along the path, respectively. The rest of the variables are as in the opaque case. With such definitions, the actual WTAR algorithm proposed for translucent networks is as follows:

1) Same as step (1) in the opaque scenario, utilizing (2a) and $(2 \mathrm{~b})$ as the metrics.

2) Select the first path as the candidate path and try to establish the connection employing a First Fit (FF) criteria for the wavelength selection, considering that the wavelength continuity constraint has to be guaranteed between regeneration spans. Please consider that wavelength continuity has also to be guaranteed between an add/drop TSP and a REG (source and destination). When regeneration is needed (the length of the path is longer than $D_{r}$ ), it will be performed in the first possible node along the end-to-end path, since we assume that every node in the network is equipped with a pool of regenerators. Depending on the length of the path, regeneration may be needed multiple times. In such cases, it will be performed in the first possible node along the accumulated path (before the accumulated path is longer than $D_{r}$ ). Additionally, we consider that any REG is capable to perform wavelength conversion.

3) Same as (3) in the opaque case.

4) Same as (4) in the opaque case.

The behavior of the translucent WTAR algorithm is the same as in the opaque case: with $\alpha=0$, the use of REGs in the network will be balanced, reducing the blocking associated to the effects of the wake-up time; with $\alpha=1$, the shortest physical routes will be selected, keeping the energy consumption low; any case in between represents a compromise solution between power savings and blocking probability.

In the following section, we evaluate the proposed algorithms through extensive simulations.

\section{TEST SCENARIO AND RESULTS}

\section{A. Scenario description}

To analyze the impact of the wake-up time $\left(T_{w}\right)$, we have executed various simulations employing two network topologies, namely, the 12-node Deutsche Telekom (DT) network [12] and the 30-node CORONET network [13]. Table I shows the main network statistics considering the shortest paths among all node pairs in the two networks to compute average values. For all the tests, we offered $4 \cdot 10^{5}$ connections to the network, which are assumed to be bidirectional and requesting one wavelength each. Connections arrive at the network following a Poisson distribution, with exponentially distributed inter-arrival times and holding times with average value IAT and HT, respective. Thus, the network load can be defined as HT/IAT. Offered traffic is uniformly distributed among all source/destination pairs. The bit-rate of the connections is $100 \mathrm{~Gb} / \mathrm{s}$, employing a 28 Gbaud PDMQPSK modulation, for which the transparent reach without regeneration is $1200 \mathrm{~km}$ [14].

For the opaque and translucent scenario, the connections are routed employing the WTAR algorithm (the specific value of the $\alpha$ parameter will be annotated for each experiment). For the transparent scenario, connections are routed employing a K-Shortest Path algorithm with physical distance as the path metric. Only routes that are shorter than the transparent reach are considered while the others are blocked. As for the wavelength assignment, a FF criteria is utilized, considering that the same wavelength must be provisioned in all links in the end-to-end path. For all three scenarios, the value of $K$ (the number of candidate paths) is equal to 6.

As a baseline scenario, we considered the DT topology network considering an opaque architecture with an ideal TSP $T_{w}$, that is, $T_{w}=0 s$. With this scenario in mind, we dimensioned the network so as to have an overall blocking probability (considering high- and low-priority traffic classes) 
TABLE I

NETWORK STATISTICS

\begin{tabular}{l|cc} 
& DT & CORONET \\
\hline Shortest path length $(\mathrm{km})$ & $48(1$ hop $)$ & $69(1 \mathrm{hop})$ \\
Longest path length $(\mathrm{km})$ & $1095(4 \mathrm{hops})$ & $7078(9 \mathrm{hops})$ \\
Average path length $(\mathrm{km})$ & 530.7424 & 3064.7195 \\
Average number of hops & 2.1363 & 4.8253
\end{tabular}

not higher than $1 \%$, assuming an offered network load of 140 and mean $\mathrm{HT}=1 \mathrm{~h}$., with a share of high and low-priority traffic of $30 \%$ and $70 \%$, respectively. As a result, we have found that nodes have to be equipped with 30 TSPs per incoming/outgoing link and the same value of wavelengths per link. The number of TSPs per node and incoming/outgoing link reserved for high-priority traffic in this basic case is $m=2$. For the sake of simplicity, we will also employ the same number of resources in the CORONET network topology. In such case, the overall blocking probability is no bigger than $2 \%$. To perform a fair comparison, although in a translucent and transparent optical network scenario TSPs are only employed in the source and destination nodes, we adopt the same number of network resources both in terms of TSPs and wavelengths as in the opaque case. This is done to avoid having the opaque scenario with much more node equipment (i.e. TSPs) than the transparent or translucent case. Moreover, for the translucent scenario we consider that each node is equipped with $N^{\prime}=15$ REGs with $m^{\prime}=1$ reserved for high-priority traffic. As a result, for this base scenario, the overall blocking probability is not higher than $1-5 \%$ of the total offered connections for both transparent and translucent cases.

On this base scenario, we analyzed the impact of the value of $T_{w}$ on the network blocking probability (BP). Since the impact of $T_{w}$ is tightly related to the traffic dynamicity (how many high-priority connections may arrive during a wakeup operation), we have investigated, for various degrees of dynamicity and values of $T_{w}$, how $\mathrm{BP}$ values of high-priority traffic evolve. To this end, we define $r=H T_{b a s e} / H T$ as the dynamicity of the traffic, denoting as $H T_{\text {base }}$ the mean HT in the base scenario (i.e., $1 \mathrm{~h}$.), modifying the mean IAT of the connections in all cases so as to maintain the same network load of 140. Hence, under the same offered load, more dynamic connections are being established.

\section{B. DT network results}

We start by evaluating the impact of the $T_{w}$ for all the three cases (opaque, translucent and transparent) considering that the routing algorithm is completely blind to the $T_{w}$, that is, no WTAR is being considered ( $\alpha=1$ in the opaque and translucent case). Fig. 3 shows the BP as a function of $r$ for high-priority traffic, considering multiple values of $T_{w}$, for the DT network topology. The base scenario $\left(T_{w}=\right.$ $0 \mathrm{~s}$ ) is also depicted as a reference. It can be appreciated that the opaque scenario is the most sensitive to the value of $T_{w}$, since, as explained before, all the end-to-end route of the connections entails the use of devices affected by the $T_{w}$. More specifically, we can see that as the $T_{w}$ increases, less traffic dynamicity can be supported without incurring into severe performance degradations. For instance, for $T_{w}=1$ min. and $r \geq 3$, the BP becomes higher than $1 \%$ while for $T_{w}=5$ min., the BP becomes higher than $1 \%$ even for the base dynamicity $(r=1)$. The translucent and transparent cases behave very similarly because, in the DT network, regeneration occurs very sparsely. Additionally, we can see that up to $T_{w}=1 \mathrm{~min}$. all the curves feature an almost flat behavior. Although not shown for the sake of space, the BP of low priority traffic is around $1-2 \%$ in the opaque case for all traffic dynamicities and $T_{w} \leq 1 \mathrm{~min}$., will it experiences a slight reduction in the case of $T_{w}=5 \mathrm{~min}$. This is due to the fact that higher values of $T_{w}$ result in more high priority connections blocked, hence, more resources can be exploited by low priority traffic.

Now let us investigate the main causes of blocking of high-priority connections. In the opaque scenario, the sole cause of blocking is due to the lack of idle TSPs, while in the translucent and opaque scenarios the vast majority of blocking (around 99\%) is due to the lack of free contiguous wavelengths. For this reason, the BP in the translucent and transparent case for $T_{w} \leq 1 \mathrm{~min}$. is higher than in the opaque case, since it is necessary to provision the same wavelength in all links along the path. Nevertheless, for a $T_{w}=5 \mathrm{~min}$., the lack of idle resources starts to be noticeable. Note however, that the experimented degradations are still much less pronounced than in the opaque case, due to the fact that less resources are affected by the $T_{w}$ (only the source and destination nodes are affected).

In light of these, we have investigated for various values of $r$ how many TSPs per node should be set to idle in order to reduce the $\mathrm{BP}$ of high-priority traffic below $1 \%$ considering a $T_{w}=1$ min. Fig. 4 depicts the obtained results (the black thick solid line marks the desirable $1 \% \mathrm{BP}$ value) for the opaque, translucent and transparent scenarios.

At least, one additional idle TSP per node should be reserved to meet the desired $\mathrm{BP}$ for $r=3,4$ and 5 in the opaque scenario. Note, however, that when more TSPs are set to idle, the $\mathrm{BP}$ of low-priority connections starts to increase, since fewer resources can be exploited by them. Hence, to maintain low-priority BP constant, a number of off TSPs equal to the additional TSPs set to idle, should be equipped in the nodes, thus increasing the overall network cost. In contrast, we can see that even increasing the number of idle TSPs does not decrease the $\mathrm{BP}$ in the translucent and transparent cases, since, as commented before, in such scenarios, the main cause of blocking is the lack of free wavelengths.

Now, we will evaluate how the blocking levels change when the routing algorithm takes into consideration the presence of the $T_{w}$. Since in the DT network the opaque scenario is the only one that is highly affected by the $T_{w}$, we will focus in the opaque case considering $\alpha=0$. To this end, Fig. 5 depicts the improvement ratio respect the case where no WTAR is performed ( $\alpha=1$ ), that is, the ratio between the $\mathrm{BP}$ in the case without WTAR and the case with WTAR. We can see that substantial improvements in the BP can be achieved with the proposed WTAR algorithm, with improvement ratios up to 100 for low values of $T_{w}$, which translates into reductions of up to 2 orders of magnitude in the BP of high priority connections. More modest improvements (around 1 order of magnitude) are achieved for higher values of $T_{w}$. Additionally, the blocking levels remain lower than $1 \%$ for all traffic dynamicities for $T_{w} \leq 1 \mathrm{~min}$. by using WTAR. This means that we can reduce the overall blocking levels without provisioning extra idle TSPs or off TSPs. Although not shown, the blocking levels of low-priority connections are 

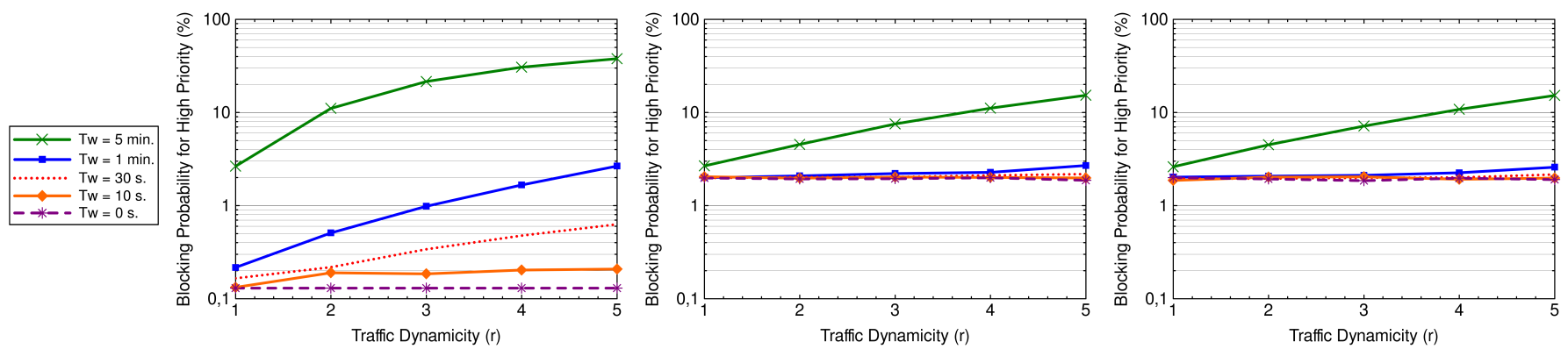

Fig. 3. Blocking as a function of $r$ for the opaque (left), translucent (middle) and transparent (right) cases for the DT network scenario without WTAR.
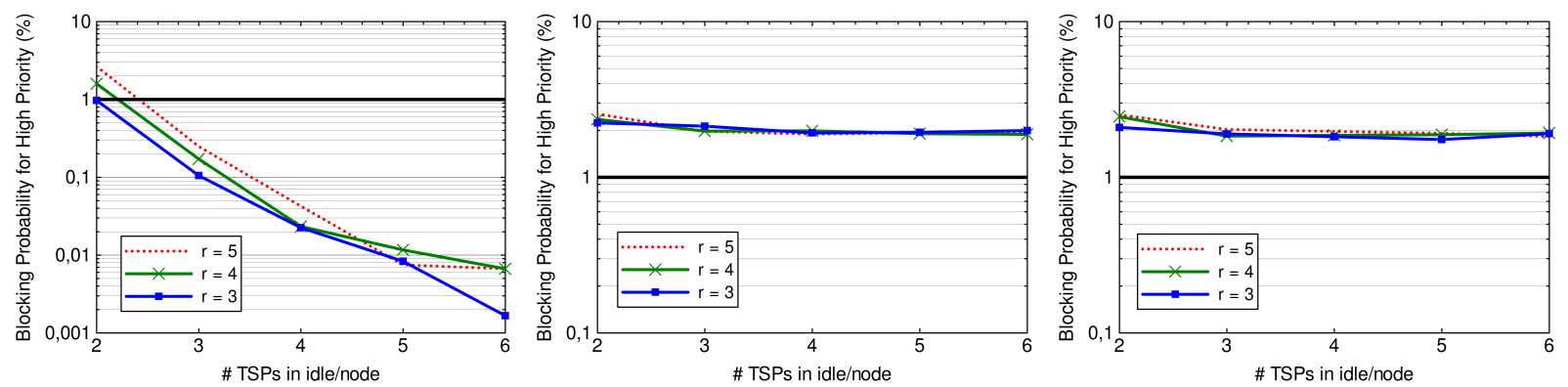

Fig. 4. Blocking as a function of the number of idle TSPs for the opaque (left), translucent (middle) and transparent (right) cases for the DT network scenario without WTAR.

also reduced (up to one order of magnitude) for all traffic dynamicities and values of $T_{w}$. This happens because, as explained before, the WTAR algorithm balances also the use of off devices in the network ensuring that there will be enough of them to perform wake-up operations. Although not shown, low priority connections also experience some improvements on their blocking figures (up to 1 order of magnitude). This is due to the fact that the routing algorithm balances the use of off devices, effectively reducing the $\mathrm{BP}$ of low priority connections.

To complete the studies in the DT network, let us now evaluate the blocking levels of high-priority traffic as a function of $\alpha$. To this end, we focus on the opaque case, considering $r=4$. Fig. 6 depicts the obtained results. We can see that the choice of $\alpha$ has a significant impact on the blocking probability. Generally, lower values of $\alpha$ result in lower blocking figures, since the load balancing term of the WTAR algorithm has more weight. This is specially evident for low values of $T_{w}$, with variations of some orders of magnitude in the blocking. As for higher values of $T_{w}$, the impact of $\alpha$ is lower due to the fact that nodes exhaust more rapidly their pool of idle resources, hence the routing algorithm has less chances to balance their utilization.

\section{CORONET network results}

At this point, let us evaluate the BP of the WTAR algorithm in a larger network topology, namely, the 30-node CORONET topology. We will focus on the opaque and the translucent cases, since the longer physical diameter of this network does not allow the establishment of transparent connections for all node pairs due to transmission reach reasons. As in the previous subsection, we start by evaluating in Fig. 7 the blocking of high-priority traffic as a function of $r$ for

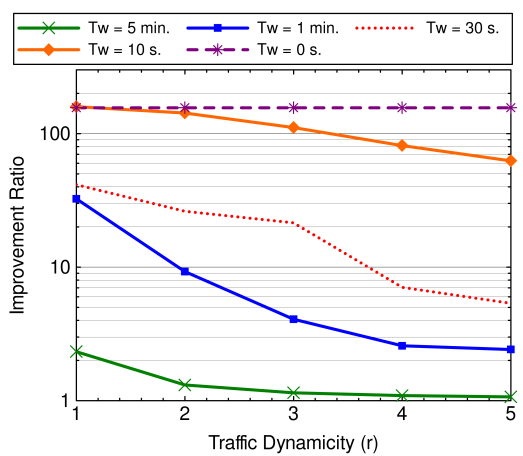

Fig. 5. Improvement ratio as a function of $r$ for high priority traffic for the DT network scenario with WTAR $(\alpha=0)$.

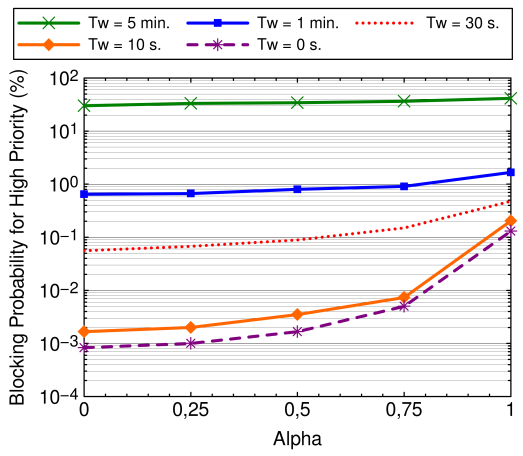

Fig. 6. Blocking as a function of $\alpha$ for the opaque case for the DT network scenario.

various values of $T_{w}$ considering that no WTAR is employed $(\alpha=1)$.

Results confirm that the opaque case is very sensitive 

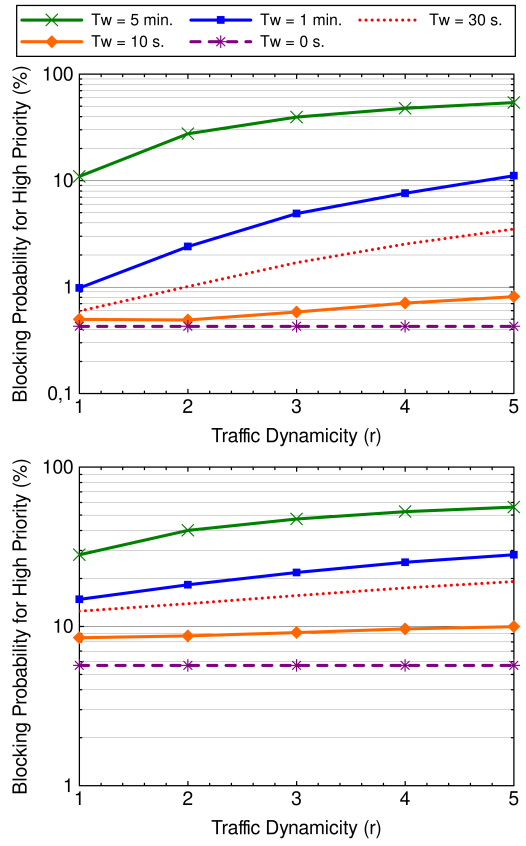

Fig. 7. Blocking as a function of $r$ for the opaque (top) and translucent (bottom) cases for the CORONET network scenario without WTAR.

to the value of $T_{w}$, allowing for less dynamic traffic as $T_{w}$ increases. As for the translucent case, we can see that in such a larger network, since more regeneration is needed, the effects of $T_{w}$ start to be noticeable already for $T_{w} \geq 10 \mathrm{~s}$. In this scenario, we observed that the main cause of blocking is the lack of idle REGs (around 60\%), whereas the second cause is the lack of free wavelengths for low values of $T_{w}$ or the lack of idle TSPs for high values of $T_{w}$. Moreover, as in the DT scenarios, as the duration of $T_{w}$ increases low priority traffic experiences a slight improvement on its blocking figures, since more resources can be exploited by it.

We will now evaluate the amount of extra idle resources (TSPs for the opaque case and REGs for the translucent case) needed to achieve a BP under $1 \%$. We consider $T_{w}=1$ min. and three dynamicity values, namely 3,4 and 5. Fig. 8 depicts the obtained results. As in the DT network scenario, the increase of idle TSPs per node in the opaque case allows reducing the blocking levels until the desired threshold is achieved, needing 1 extra idle TSP for $r=3$ and 4 , and 2 for $r=5$. In the translucent case, even though increasing the number of idle REGs per node reduces the blocking, the reductions do not allow to achieve the targeted performance without substantial increases on the reserved resources. For example, it would be necessary to provision at least 6-7 extra idle REGs per node to achieve a BP below $1 \%$.

Let us now evaluate the BP for the WTAR algorithm with $\alpha=0$. As in the DT case, we will depict the improvement ratio respect the case without WTAR. Fig. 9 depicts the obtained results. We can see that more moderate reductions are achieved when compared to the DT network. This is due to the fact that in the CORONET network, since connections go through a larger number of devices, the overall blocking figures are higher due to multiple nodes simultaneously running out of idle devices. In such situation, the routing algorithm has less chances to influence on the utilization of the $\mathrm{OE}$ devices by means of load balancing. Nevertheless,
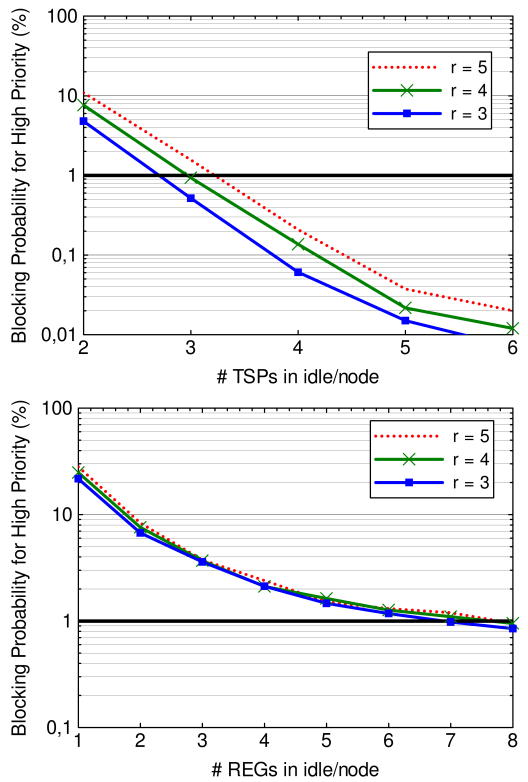

Fig. 8. Blocking as a function of the number of idle resources for the opaque (top) and translucent (bottom) cases for the CORONET network scenario without WTAR.

reductions in the blocking up to around $70 \%$ can be achieved in some cases. Like in the DT scenario, low priority connections also experience some reduction on their blocking figures, since the routing algorithm also balances the use of off devices. Next, we evaluated the evolution of the blocking probability as a function of $\alpha$ for both opaque and translucent network scenarios, fixing $r=4$. Figure 10 depicts the obtained results. It can be seen that the impact of $\alpha$ in the CORONET network is less acute when compared to the DT network, due to the fact that connections are experiencing more blocking. Nevertheless, it can be seen that lower values of $\alpha$ generally entail lower blocking figures, even for high values of $T_{w}$.

To conclude our study, we have evaluated the average daily power consumption under the fluctuating daily traffic profile presented in [15] considering three cases: 1) all devices stay always powered-on, like in traditional WDM networks; 2) a sleep-mode strategy is adopted but the routing algorithm is blind to the effects of $\left.T_{w}(\alpha=1) ; 3\right)$ a sleep-mode strategy is adopted and the presented WTAR algorithm is used $(\alpha=0)$. We use the CORONET network topology, considering both an opaque and translucent architectures. Additionally, we set $T_{w}=1 \mathrm{~min}$. and $r=4$. For the sake of fairness, in the all-on scenario, the same dimensioning used to get the other results has been assumed. Moreover, for the cases where sleepenabled devices are employed, nodes are equipped with extra idle and off devices per node whenever necessary according to the results presented previously. We assume the same power figures for the devices as in [4].

In order to present a fair comparison between the three cases and decouple the power consumption from the blocking figures, we define the normalized power consumption as the ratio of the average total power consumption in the network divided by the total number of accepted connections. Utilizing this metric, Fig. 11 shows the evolution of the power consumption along the day for the three cases. It can be appreciated that huge savings (around 70\% during low 

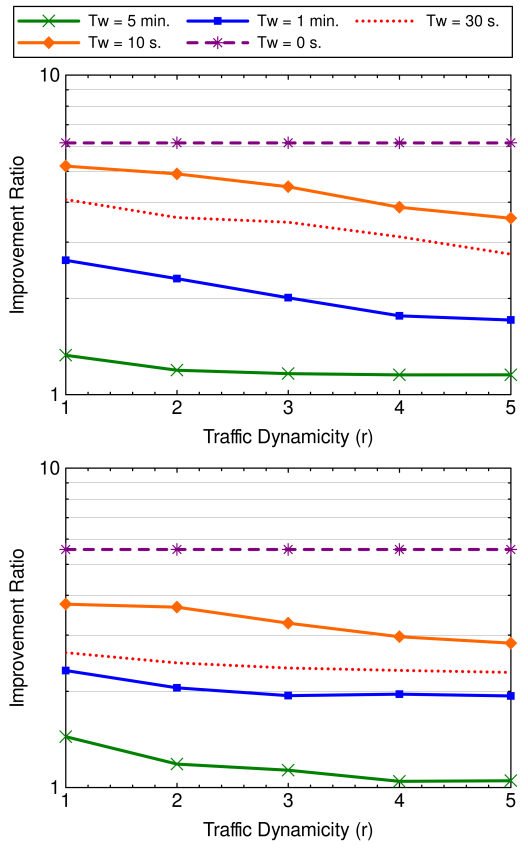

Fig. 9. Improvement ratio as a function of $r$ for high priority traffic for the opaque (top) and translucent (bottom) cases for the CORONET network scenario with WTAR $(\alpha=0)$.
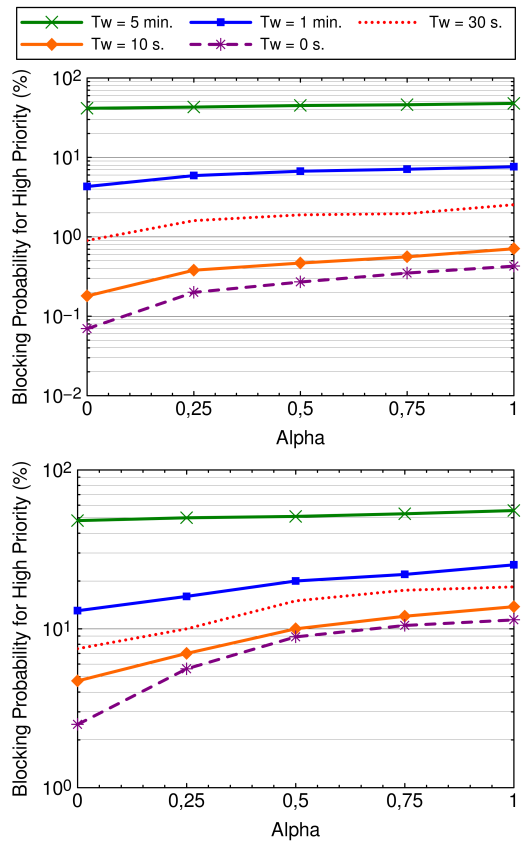

Fig. 10. Blocking as a function of $\alpha$ for the opaque (top) and translucent (bottom) cases for the CORONET network scenario.

traffic periods and around $30-40 \%$ in average ) are attained when the proposed power management strategy is employed. Focusing on the differences between employing or not the proposed WTAR algorithm, we can see that the WTAR algorithm results in higher average power figures. This is basically due to the fact that with the WTAR algorithm more connections are accepted, so more power is consumed. Additionally, as the WTAR algorithm balances the use of the network elements (TSPs and REGs), slightly longer paths are employed, increasing the number of devices that need
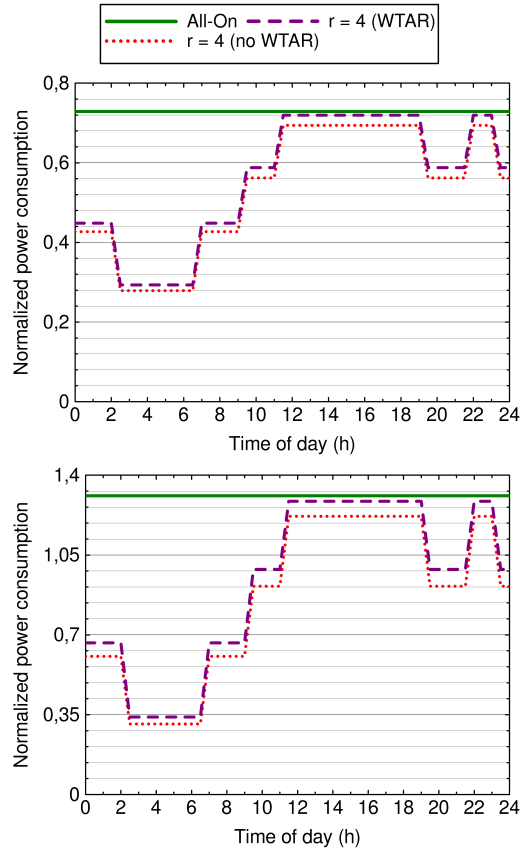

Fig. 11. Normalized daily power consumption for the opaque (top) and translucent (bottom) cases in the CORONET network

to be powered-on. Nevertheless, this increase in power when compared against the case without WTAR is very small and gets compensated with the substantial reductions in the BP that can be achieved (up to 2 orders of magnitude) and the fact that, under certain conditions, it is not required to equip network nodes with extra devices, allowing for a lower network CAPEX.

\section{CONCLUSiON}

In this work, we evaluated the impact of the wake-up time of transponders and regenerators in multi-service core optical network employing sleep-mode enabled devices. We showed that various ranges of degradations can happen depending on the type of optical network (transparent, translucent and opaque), the duration of the wake-up operation of the optical devices, the dynamicity of the traffic and the geographical size of the network. Particularly, we showed that opaque network are the most sensitive to degradations due to long wake-up time operations, due to the fact that the whole end-to-end path composing a connection is affected.

To compensate such effects, we proposed a novel routing algorithm called Wake-up Time Aware Routing (WTAR) that aims to balance the use of idle resources on the network so as to reduce the blocking associated to the lack of them. We showed that significant reductions on blocking can be achieved with the proper WTAR algorithm (up to 2 orders of magnitude in the opaque case and up to $70 \%$ in the translucent case), with the added benefit that such reduction can be achieved with very small increments, or no increments at all, in the equipment amount per network node, maintaining the overall network cost low.

Additionally, we showed that substantial reductions can be achieved (up to 70\%) in the average daily network power consumption when sleep-mode enabled transponders and regenerators are employed when compared to traditional WDM network scenarios. The additional power consumption that 
entails the use of the WTAR algorithm gets compensated by the significant reductions in connection blocking and network CAPEX when compared with a routing algorithm that is completely blind to the effects of the wake-up time.

\section{ACKNOWLEDGMENT}

The work on this paper has been funded by Spanish Science Ministry through the project ELASTIC (TEC201127310) and the GreenTouch consortium.

\section{REFERENCES}

[1] C. Lange, D. Kosiankowski, R. Weidmann, A. Gladisch, "Energy Consumption of Telecommunication Networks", Proc. of ECOC 2009 , Sept. 2009.

[2] L. Wosinska, A. Jirattigalachote, P. Monti, A. Tzanakaki, K. Katrinis, "Energy efficient approach for survivable WDM optical networks", Proc. of ICTON 2010, July 2010.

[3] Y. Zhang, P. Chowdhury, M. Tornatore, B. Mukherjee, "Energy Efficiency in Telecom Optical Networks", IEEE Comm. Surveys and Tutorials, vol. 12 , no. 4, pp. 441-458, July 2010.

[4] A. Morea, S. Spadaro, O. Rival, J. Perelló, F. Agraz, D. Verchere, "Power management of optoelectronic interfaces for dynamic optical networks", Proc. of ECOC 2011, We.8.K.3, Sept. 2011.

[5] A. Muhammad, P. Monti, I. Cerutti, L. Wosinska, P. Castoldi, A. Tzanakaki, "Energy-Efficient WDM Network Planning with Dedicated Protection Resources in Sleep Mode", Proc. of GLOBECOM 2010, Dec. 2010

[6] I. Cerutti, N. Sambo, P. Castoldi, "Sleeping Link Selection for Energy-Efficient GMPLS Networks", IEEE/OSA Journal of Lightwave Technology, vol. 29, no. 15, pp. 2292-2298, June 2011.

[7] A. Morea, J. Perelló, F. Agraz, S. Spadaro, "Demonstration of GMPLS-controlled device power management for next generation green optical networks", Proc. of OFC/NFOEC 2012, OM3G.6, March 2012.

[8] F. Musumeci, M. Tornatore, M. Riunno, A. Pattavina, "Blocking analysis for green WDM networks with transponder power management", Proc. of ICTON 2013, We.A4.5, June 2013.

[9] A. Pagès, M. Tornatore, J. Perelló, S. Spadaro, A. Morea, "Impact of transponders and regenerators wake-up time on sleep-mode enabled translucent optical networks", Proc. of OFC/NFOEC 2014, W4B.4, March 2014

[10] N. Sambo, P. Castoldi, F. Cugini, G. Bottari P. Iovanna, "Toward high-rate and flexible optical networks", IEEE Communications Magazine, vol. 50, no. 5, pp. 66-72, May 2012.

[11] M.-Y. Liao, M.-Y. Luo, C.-S. Yang, C.-H. Chen, P.-C. Wu, Y.-C Chen, "Design and evaluation of deep packet inspection system: A case study", IET Networks, vol. 1, no. 1, pp. 2-9, March 2012.

[12] J. Perelló, A. Morea, S. Spadaro, A. Pagès, S. Ricciardi, M. Gunkel, G. Junyent, "Power consumption reduction through elastic data rate adaptation in survivable multi-layer optical networks", Photonic Net. Comm., June 2014.

[13] Monarch Network Architects, http://monarchna.com/topology.html.

[14] A. Klekamp, R. Dischler, F. Buchali, "Transmission Reach of Optical-OFDM Superchannels with 10-600 Gb/s for Transparent Bit-Rate Adaptive Networks", Proc. of ECOC 2011, Tu.3.K.2, Sept. 2011.

[15] A. Morea, O. Rival, N. Brochier, E. Le Rouzic, "Datarate Adaptation for Night-Time Energy Savings in Core Networks", IEEE / OSA Journal of Lightwave Technology, vol. 31, no. 5, pp. 779-785, March 2013.

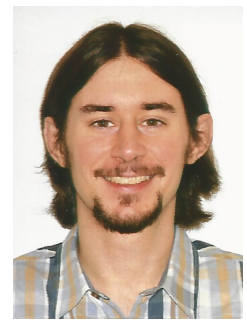

Albert Pagès received the M.Sc. and the Ph.D. degrees in Telecommunications Engineering in 2010 and 2014, respectively, both from Universitat Politècnica de Catalunya (UPC), Spain. He is currently working as a postdoctoral researcher in the Optical Communication Group (GCO) and the Advanced Broadband Communications Center (CCABA) of UPC. He has participated in various FP-7 European research projects (DICONET, GEYSERS, STRONGEST, LIGHTNESS, etc.). His research interests include optimization and management issues in elastic optical networks as well as virtualization techniques in alloptical networks and data center environments.

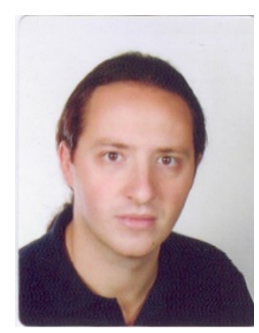

Massimo Tornatore received a Ph.D. degree in Information Engineering in 2006 from Politecnico di Milano. He is currently working as an assistant professor in the Department of Electronics, Information and Bioengineering at Politecnico di Milano. He also holds an appointment as adjunct associate professor in the Department of Computer Science at the University of California, Davis, where he served as a postdoc researcher in 2008 and 2009. He is author of more than 180 conference and journal papers and his research interests include design, protection and energy efficiency in optical core, metro and access networks. He is a co-recipient of 6 Best Paper Awards from IEEE conferences.

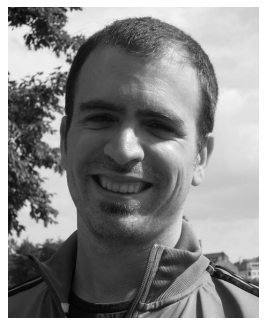

Jordi Perelló received the M.Sc. and Ph.D. degrees in telecommunications engineering in 2005 and in 2009 , respectively, both from the Universitat Politècnica de Catalunya (UPC), Spain. Currently, he is an Assistant Professor in the Department of Computer Architecture (DAC) at the UPC. He has participated in various FP6 and FP7 European research projects (LIGHTNESS, EULER, STRONGEST, DICONET etc.). Dr. Perelló has published more than 70 articles in international journals, conference proceedings and book chapters. His research interests concern the resource management, quality of service, survivability and virtualization of next-generation optical transport networks.

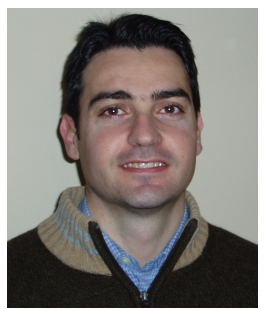

Salvatore Spadaro received the M.Sc. (2000) and the Ph.D. (2005) degrees in Telecommunications Engineering from Universitat Politècnica de Catalunya (UPC), Spain. He also received the Dr. Ing. degree in Electrical Engineering from Politecnico di Torino (2000), Italy. He is currently Associate Professor in the Optical Communications group of the Signal Theory and Communications Dept. of UPC. Since 2000 he is a staff member of the Advanced Broadband Communications Center (CCABA) of UPC. He has participated in various FP-6 and FP-7 European research projects. Dr. Spadaro has published more than 100 articles in international journals and conference proceedings. His research interests are in the fields of all-optical networks with emphasis on network control and management, resilience and network virtualization. 


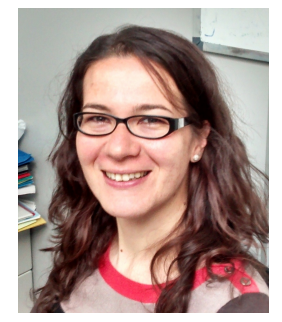

Annalisa Morea is a research engineer at Bell Labs France. She graduated in telecommunication from Politecnico di Torino, Italy, and from École Nationale Supérieure des Télécommunications (ENST) Paris, France. She received a Ph.D. degree in computer science and networks from ENST Paris, carrying out her work at the laboratories of France Telecom R\&D in Lannion. Her thesis focused on optical network communication, particularly on the performance and interests of new translucent optical networks. She has participated in various $\mathrm{Na}-$ tional and European research projects. She is also involved in the GreenTouch initiative for improving the network energy consumption. Her research interests are in the fields of optical networks with emphasis on multilayer and elastic optical routing to innovate the optical network solutions. 